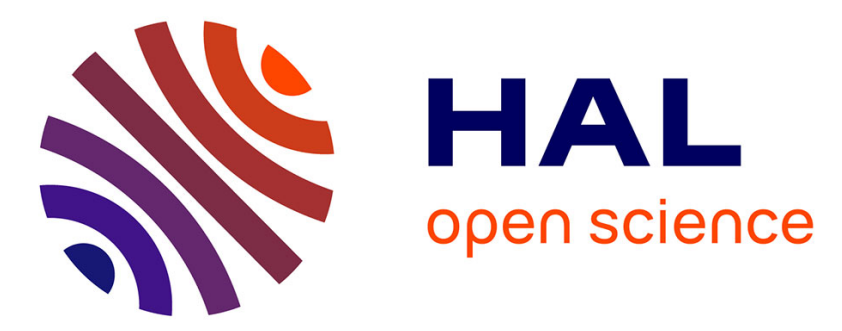

\title{
An approach of human driving behavior correction based on Dynamic Window Approach
}

Yue Kang, Danilo Alves de Lima, Alessandro Corrêa Victorino

\section{To cite this version:}

Yue Kang, Danilo Alves de Lima, Alessandro Corrêa Victorino. An approach of human driving behavior correction based on Dynamic Window Approach. IEEE Intelligent Vehicles Symposium (IV 2014), Jun 2014, Dearborn, Michigan, United States. pp.304 - 309, 10.1109/IVS.2014.6856543 . hal-01120337

\section{HAL Id: hal-01120337 \\ https://hal.science/hal-01120337}

Submitted on 25 Feb 2015

HAL is a multi-disciplinary open access archive for the deposit and dissemination of scientific research documents, whether they are published or not. The documents may come from teaching and research institutions in France or abroad, or from public or private research centers.
L'archive ouverte pluridisciplinaire HAL, est destinée au dépôt et à la diffusion de documents scientifiques de niveau recherche, publiés ou non, émanant des établissements d'enseignement et de recherche français ou étrangers, des laboratoires publics ou privés. 


\title{
An approach of human driving behavior correction based on Dynamic Window Approach
}

\author{
Yue Kang, Danilo Alves de Lima, Alessandro Corrêa Victorino
}

\begin{abstract}
This paper presents the approach of an applicable safety driving methodology for human drivers based on Dynamic Window Approach (DWA), as an implementation of Advanced Driving Assist Systems (ADASs). The human driving behaviors are modelled for the design of controller, refined by referential paths using evasive trajectory model, and the linear and angular velocities are limited and corrected by DWA which performed as an obstacle avoidance strategy. Results of trajectory following and obstacle avoidance are compared with the visual servoing (VS) controller as a corresponding approach of autonomous control pattern.
\end{abstract}

Index Terms-ADAS, Human Driving Behavior, Driving Safety, Dynamic Window Approach

\section{INTRODUCTION}

Safety has been of major and critical interest to the automation systems since the dawn of the automotive industry. Developing effective active safety devices has gained emphasis within the last decades for accident prevention and harm reduction [1]. A large number of road accidents occur on the main and secondary roads each year, and driver error is one of the reasons with evident importance [2]. A growing number of applicable approaches has been brought out in the progress of various modelings of driving behavior. For instance, a similar system is proposed in [3] which takes into account vehicle dynamics and driver behavior as coefficient that express how much acceleration the driver could accept. Another approach of software-based collision avoidance system was implemented to a dedicated shortrange vehicle-to-vehicle communication for better mitigating accident collisions [4]. More recently in [5] a safety-based approaching behavioural model was proposed for the driver's car-following behavior with various driving characteristics, deducted from an integrated driving behavior modeling framework in previous works [6] [7].

However, these achievements focusing on modeling the characteristics and behavior of intelligent vehicles rarely employ the driver's control instructions to the vehicles. Taking a step backwards, fully autonomous vehicles are currently available in rather ideal and constrained experimental environment, and human effect during the driving has failed to be taken into sufficient consideration while human drivers' repulsive controlling characteristics are still major causes of road crashes [8]. Consequently, approaches of applicable detection and correction for human decision

The authors are with Heudiasyc UMR CNRS 7253 Université de Technologie de Compiègne. Yue Kang holds a Ph.D scholarship from Chinese Scolarship Council and Picardi region in France. Contact authors yue.kang@hds.utc.fr faults, as a supplementary driving assistance system, are in extreme need.

Traditional Advanced Driver Assistance Systems (ADASs) have been exceedingly advanced over the past few decades [2]. From the first generation described by "lateral and longitudinal stabilization and control" and represented by the renowned Anti-lock Braking System (ABS) and the Electronic Stability Control (ESC) system, to the later "careful active actions and warnings" generation with the Adaptive Cruise Control (ACC) [9], Lane Departure Warning (LDW) [10] and Lane Keeping Support (LKS) systems [11] [12], till the last generation characterized by "warnings and full active actions" for which the Mitigation to effects of Collisions (CM) or Collisions Avoidance (CAV) [8] by emergency braking or steering holds the reputation, the ADASs have been playing without doubt a rewarding and efficient role in the autonomous driving industry. Our work continues in the same directions leaded by previous approaches while emphasizing human drivers' controlling characteristics.

As a complement of ADAS with focus on the judgement and correction of human drivers' behavior, our approach possesses a close relation with teleoperation systems. In [13] a detailed survey of bilateral teleoperation is performed along with interactions between human operators, masterslave robotic servo systems and the operation environment. In more recent works as in [14] and [15], haptic robotic systems interact with human users by force feedback and tactile sensation feedback such that operators amend and correct their actions in teleoperation. Quite a few related approaches harbor the significance on operational humanrobot interactions, which is also acknowledged in the domain of ADAS as human-vehicle interactions. In our work, this interaction, which is performed as the correction of the human drivers' decision faults, is of essential importance.

The objective of our approach is to simulate and correct the human drivers' decision faults, which is accomplished by applying the Dynamic Window Approach (DWA) [16]. The human decision faults are modelled as a Human Driving Behavior (HDB) controller with predefined trajectory paths where potential collisions and crashes occur. This approach is emerged from the approach of our previous work [17], where the DWA method was applied with an image-based Visual Servoing (VS) controller [18] to perform the autonomous road lane following task while avoiding obstacles. A detailed explanation of this method can be referred in the other paper submitted by the same authors. While working outstandingly for the trajectory navigation tasks, the VS control fails to 


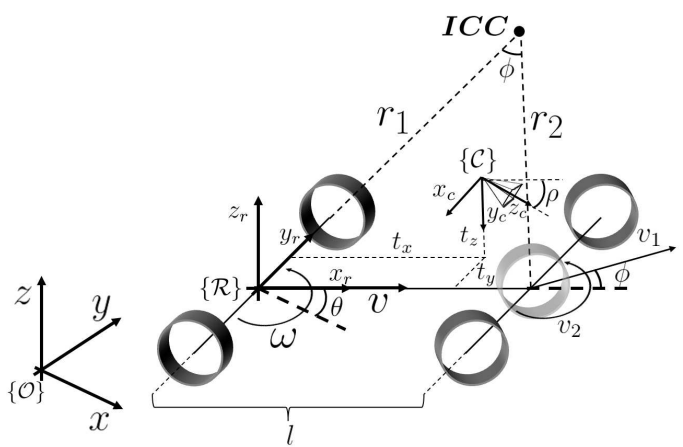

Fig. 1. Cinematic model diagram for a front wheel car-like robot. In this model the vehicle reference frame $\mathcal{R}$ performs circular trajectories related to the instantaneous center of curvature (ICC). The pinhole camera frame is also represented in $\mathcal{C}$.

guarantee the safety of driving with the lack of obstacle avoidance. In the current approach we focus on the correction of the human decision faults in the same driving environment of the autonomous VS-DWA cooperative control pattern, and a comparaison of performance between the two controllers.

In order to present this methodology, this article is organized as follows: in Section II, we present the robot model and the simulation environment; in Section III, we illustrate our design of control pattern in the perspective of humanvehicle interactions with a short introduction of DWA-VS controller used for the comparaison, and the evasive trajectory as the reference for the design of HDB controller; finally experimental results with performance analysis are presented in section IV.

\section{General Definitions}

The robot in this work is considered to move in a planar workspace, similar to the one described in [18], where the road lane center defines a path once differentiable in $\mathbb{R}^{2}$. The robot follows the path according to the kinematic model of a front wheel car [19] as:

$$
\left[\begin{array}{c}
\dot{x_{r}} \\
\dot{y_{r}} \\
\dot{\theta} \\
\dot{\phi}
\end{array}\right]=\left[\begin{array}{c}
\cos \theta \cos \phi \\
\sin \theta \cos \phi \\
\sin \phi / l \\
0
\end{array}\right] v_{1}+\left[\begin{array}{l}
0 \\
0 \\
0 \\
1
\end{array}\right] v_{2},
$$

where the vehicle configuration is given by $q=\left[x_{r} y_{r} \theta \phi\right]^{T}$, with the position $\left(x_{r}, y_{r}\right)$ and orientation $(\theta)$ of the car's reference frame $\{\mathcal{R}\}$ in relation to a static world reference frame $\{\mathcal{O}\}$, and $\phi$ is the average steering angle of each front wheel by the Ackerman's approximation. The orientation and steering angles $(\theta$ and $\phi)$ are positive counter-clockwise, with $\theta \in]-\pi, \pi]$ and $\phi \in\left[-\phi_{\max }, \phi_{\max }\right]$. The variables are illustrated in the Figure 1.

The control input for the vehicle of the model (1) is $u=$ $\left[\begin{array}{ll}v_{1} & v_{2}\end{array}\right]^{T}$, which consists of respectively the linear velocity $v_{1}$ and the steering velocity $v_{2}$ of the front wheels. With the relationship between linear velocity $v$ and front wheels velocity $v_{1}$ as $v=v_{1} \cos (\phi)$, as well as the angular velocity $\dot{\theta}=v_{1} \cos (\phi) / r_{1}=\omega$ and the steering angle, it is possible to choose the robot control input as $u_{r}=[v \omega]^{T}$.

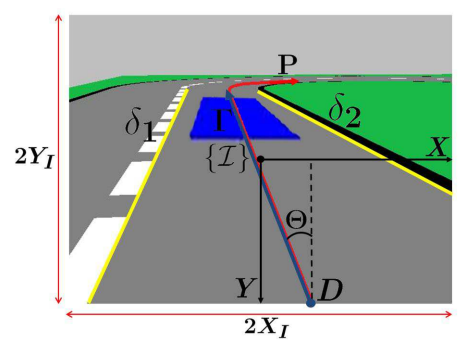

Fig. 2. Image frame $\{\mathcal{I}\}$ with the road lane center projection $P$ (in red) related to the boundaries $\delta_{1}$ and $\delta_{2}$ (in yellow), its tangent $\Gamma$ (in dark blue) at the point $D$ and the angle offset $\Theta$ of $\Gamma$ to the axis $-Y$.

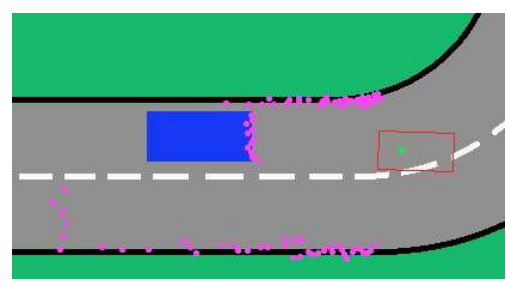

Fig. 3. Obstacles represented in world frame $\{\mathcal{W}\}$ (in blue), with detection of obstacles and also navigable zone boundaries (in pink).

For the presentation of Visual Servoing (VS) control used for further comparaisons in the Section III and IV, the Figure 1 also represents the camera frame $\{\mathcal{C}\}$ with optical center position in $\left(x_{c}, y_{c}, z_{c}\right)=\left(t_{x}, t_{y}, t_{z}\right)$ in the robot frame and a constant tilt offset $0<\rho<\frac{\pi}{2}$ related to the $x_{r}$ axis. The camera's image frame $\{\mathcal{I}\}$ is represented in the Figure 2, with defined size as $\left(2 X_{I}, 2 Y_{I}\right)$.

The obstacles on the road are represented as blocks with similar size to our vehicle, as in the Figure 3. An example from the camera point of view is also represented in the Figure 2. To simplify the problem, we assume that the obstacles are in two dimensions, rectangle-shaped and can be detected by exteroceptive sensors, for instance, LIDAR. This assumption helps reduce the difficulty of establishing the simulating and experimental environment while maintain the feasibility and reliability of the obstacle avoidance required by the Dynamic Window Approach (DWA).

\section{Human-VEhicle InTERACTIONS}

Recalling the principal aim of our work, we propose an autonomous correction mechanisme to the driving behaviors based on the Dynamic Window Approach (DWA). With the interaction between human drivers and the vehicle, the driving behaviors which are represented by the vehicle configuration $q=\left[\begin{array}{lll}x_{r} & y_{r} & \theta\end{array}\right]^{T}$ defined in the Section II, are revised and optimized by the application of the new control input $u=\left[\begin{array}{ll}v_{1} & v_{2}\end{array}\right]^{T}$ calculated by DWA.

\section{A. Dynamic Window Approach}

The Dynamic Window Approach is a reactive obstacle avoidance technique proposed originally by [16], with a modification for car-like robots presented in [20], This approach optimize an objective function (2) in order to select the best control input regarding the desired configuration 
to the robot. The objective fonction is constituted with three weighted components: the goal position (heading), the obstacle distance (dist) and the final linear velocity (velocity), represented as:

$$
\begin{aligned}
D W A(v, \omega)= & \alpha \cdot \operatorname{heading}(v, \omega)+\beta \cdot \operatorname{dist}(v, \omega) \\
& +\gamma \cdot \operatorname{velocity}(v, \omega) .
\end{aligned}
$$

Initially, for the actual velocity of the robot $\left(v_{a}, \omega_{a}\right)$, the dynamic window $V_{d}$ is defined for all reachable velocities in a time interval $\triangle t$ as:

$$
\begin{aligned}
V_{d}=\{(v, \omega) \mid v & \in\left[v_{a}-\dot{v} \triangle t, v_{a}+\dot{v} \triangle t\right], \\
\omega & \left.\in\left[\omega_{a}-\dot{\omega} \triangle t, \omega_{a}+\dot{\omega} \triangle t\right]\right\}
\end{aligned}
$$

where $\dot{v}$ and $\dot{\omega}$ are respectively the linear and angular acceleration of the robot.

Following, each reachable velocity must be classified as admissible or not depending on the obstacle distance (dist) and the robot maximum breaking accelerations $\left(\dot{v}_{b}, \dot{\omega}_{b}\right)$. The velocity is guaranteed as admissible if the distance to the obstacle in a circular trajectory exceeds the distance required to stop safely the vehicle. The collision detection algorithm implemented in the function $\operatorname{dist}(v, \omega)$ is the one proposed by [21] for polygonal robots, which defines the set of admissible velocities:

$$
\begin{aligned}
V_{a}=\{(v, \omega) \mid v & \leq \sqrt{2 \cdot \operatorname{dist}(v, \omega) \cdot \dot{v}_{b}}, \\
\omega & \left.\leq \sqrt{2 \cdot \operatorname{dist}(v, \omega) \cdot \dot{\omega}_{b}}\right\} .
\end{aligned}
$$

Finally, $V_{s}$ is defined to satisfy the maximum acceleration constraints $\dot{v}_{\max }$ and $\dot{\omega}_{\max }$. The dynamic window search space, which considers the actual speed of the vehicle, its accelerations, the obstacles in the workspace, and also the physical limits of the vehicle is consequently generated as:

$$
V_{D W}=V_{d} \cap V_{a} \cap V_{s} .
$$

\section{B. Design of Control Pattern}

According to the control input for the vehicle defined in the Section II as $u=\left[\begin{array}{ll}v_{1} & v_{2}\end{array}\right]^{T}$, we simplify the human control pattern. In an ideal driving environment where no obstacles are involved, the obstacle distance (dist) in the objective function is omitted. Naturally human driver conducts the vehicle in the center of the road lanes, with which the goal position (heading) in the objective function 2 is determined. The linear and the angular speeds depend on the maximum velocity constrain of the vehicle, the legal speed limitions of the road and the road conditions (turnings, intersections, etc.). In a simplified experimental circle track, the linear velocity $v_{1}$ and the steering velocity $v_{2}$ are constrained respecting these elements.

In general, human drivers avoid the collision with two principal reactions: decelerating and turning. Without any auxiliary perception of the environment, these reactions depend only on the individual judgement of the human drivers [22]. Consequently, the DWA guarantees a limitation of the input velocities with the help of the obstacle avoidance and the implement of the obstacle distance (dist). Nonetheless, apart form the obstacle avoidance, the essential aim of human drivers is still guiding the vehicle in the center of the road, and the the goal position (heading) remains unchanged.

As a contrast to the human control pattern, we apply the Visual Servoing (VS) strategy carried out by Cherubini et al [18], in which two non-linear feedback controllers are designed for the propose of following a line path projected in the camera image. With the absence of the capacity to guarantee the safety of driving, a correction by an obstacle avoidance technique, in this case the DWA, is consequently necessary. Omitting the detail of visual perception and projection, the design of the feedback control of VS is represented as:

$$
\omega=-B_{c}^{+}\left(\lambda e+A_{c} v_{d}\right)
$$

where $\omega$ is the feedback gain of the controller, $e$ is the state error calculated according to the visual characteristics from the projected image, $A_{c}$ and $B_{c}$ are matrixes from the kinematic model (1) transformed to the image frame, and $v_{d}$ is the desired linear velocity of the tracking control. For more details of the implementation and stability analysis see [23].

To imitate the behavior of human driving, we propose an ordinary but practicable design of feedback control according to the Euclidean distance between the position $\left(x_{r}, y_{r}\right)$ of the vehicle and a predefined referential trajectory path which represents the desired driving route. This design of feedback control, namely human driving behavior (HDB) controller is represented as:

$$
\omega=\frac{v_{d}}{v_{l}} \sqrt{\left(x_{r}-x_{p}\right)^{2}+\left(y_{r}-y_{p}\right)^{2}}+\beta,
$$

where $\omega$ is the feedback gain of the controller, $v_{d}$ is the desired linear velocity of the tracking control, $v_{l}$ is the velocity limitation of the vehicle, $\left(x_{r}, y_{r}\right)$ are coordinates of the position of the vehicle, $\left(x_{p}, y_{p}\right)$ are coordinates of the corresponding point on the referential path, and $\beta$ is an additive white Gaussian noise (AWGN) which represents inaccuracy of human drivers' control. Noted that this controller involves no angular information. This omission is acceptable in the circumstance where human drivers are rarely aware of the precise drift angle of the vehicle [24].

\section{Referential Path Modeling}

For the requirement of predefinition of the referential path in the design of HDB controller, a mathematical model of driving trajectory is essential. From the approach of vehicle trajectory prediction [25] we are confirmed that, in an appropriately simplified driving circumstance, modeling the human drivers' behaviors with an ensemble of data, including the vehicle position coordinates which consist of a discrete path, and the linear and angular velocities representing the decision of acceleration, brake and steering, is practical and adequate. In an obstacle-free circumstance this path is located in the middle of the drivable road, in the form of segments in a straight line or an arc, depending on the road driving condition characteristics. With obstacles involved, this geometrical layout of driving path is inadequate. In our 
approach, an evasive trajectory [8] is applied in the task of obstacle avoidance. This trajectory is described by a sigmoid curve in the form as:

$$
y(x)=\frac{y_{m}}{1+e^{-a(x-c)}},
$$

where $y_{m}$ is the maximum $y$-value, $a$ defines the slope at $x=c$ of the sigmoid curve and $c$ defines the position of the inflection point of the curve, therefore the total length as $s=2 c$. The curve of the trajectory along with its parameters is presented in the Figure 4.

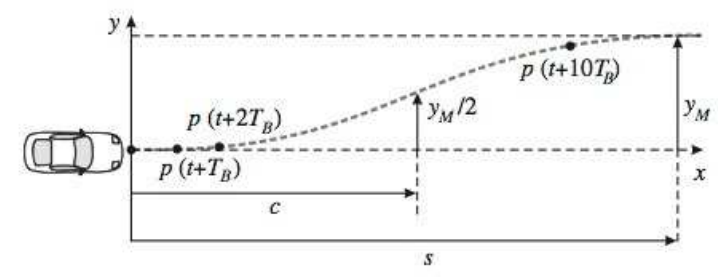

Fig. 4. Evasive trajectory for obstacle avoidance.

Noted that the definition of the evasive trajectory is not a function of time (i.e. an abuse of the naming "trajectory"), we utilize henceforth this model as a segment of a referential path. For the determination of the referential path in the task of obstacle avoidance for HDB controller, we manually revise the initial path, which is obtained in the obstacle-free circumstance, at the segments where obstacles occupy the initial path, as presented in the Figure 5.

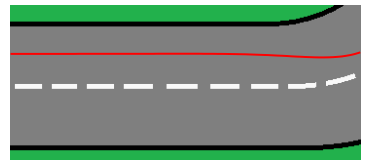

(a) Initial referential path.

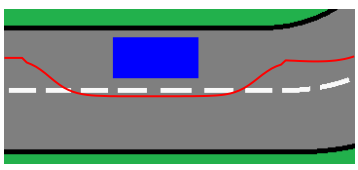

(b) Revised referential path with obstacles.
Fig. 5. Referential path revision using evasive trajectory.

\section{EXPERIMENTAL RESULTS}

\section{A. Validation}

Our first experiment aims at validating the design of imitational feedback controlling mechanism of the HDB controller that mimics the driving behaviors of humankind. The vehicle is set in a closed-loop athletic-track-shaped environment as workspace without obstacles, in which a referential clockwise path in the middle of the right-side road is predefined. The vehicle succeeded the path following task in accordance with the HDB controller applied to the kinematic model (1), as represented in the Figure 6.

The results in the Figure 6(a) demonstrated the driving route by HDB controller with an ideal referential path, while the Figure 6(b) illustrated the divergence of the coordinates of the actual driving route in comparison with the reference. While the overall route converged to the referential path, the largest deviation of the driving route occurred when the

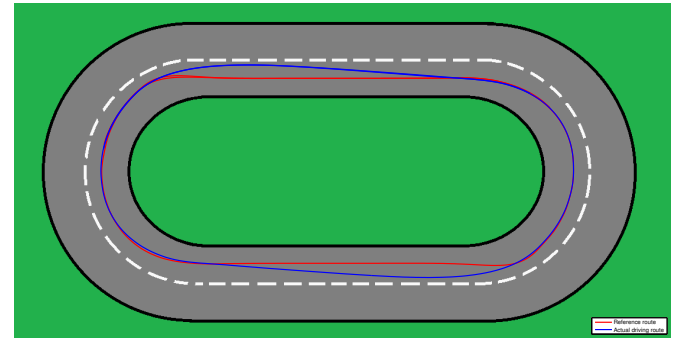

(a) Reference path (in red, hereinafter) and actual driving route by HDB controller (in blue, hereinafter).

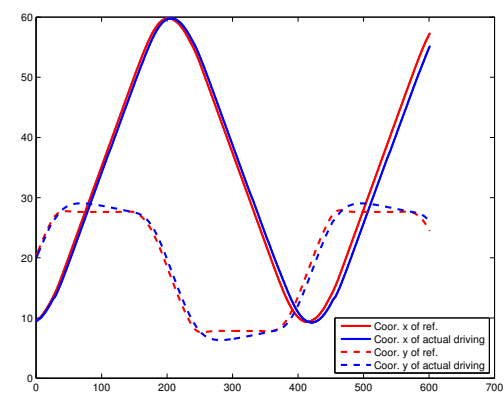

(b) Contrast of coordinates $\mathrm{X}$ and $\mathrm{Y}$ of the two routes.

Fig. 6. Validation of HDB controller design.

vehicle left the curve and entered the straight road. This phenomenon is due to the absence of angular information in the design of the HDB controller, which causes time delay of establishing the steady angular state. It also reflects the common failure of the judgement by the human drivers as incorrect estimation of the actual steering angle of the vehicle.

\section{B. Comparison with VS}

For the purpose of proving the feasibility and reliability of our methodology in the sense of safety driving, we set up obstacles showed in the Figure 3 in the simulation environment, and apply DWA method to both HDB and VS controllers. The results are presented in the Figure 7.

The routing result presented in the Figure 7(a) demonstrated that both controllers succeeded in guiding the robot to avoid obstacles with the application of DWA. Although the difference between the two routes did not prove adequately the advantage of our approach, the Figure 7(b) demonstrated that, after an essential deceleration before engaging the avoidance, the vehicle applied with the HDB controller passed the obstacle while maintaining a relatively high linear velocity. In contrast, with a sharp drop followed by a long period of low-speed forwarding, the robot guided by the VS controller failed to retain the velocity during a passing manoeuvre, which demanded a consequently long passing time and an increase of energy consuming.

In addition, the Figure 7(c) illustrated that HDB controller guaranteed the smoothness of turning procedure. The angular velocity of the robot calculated by HDB controller remained 


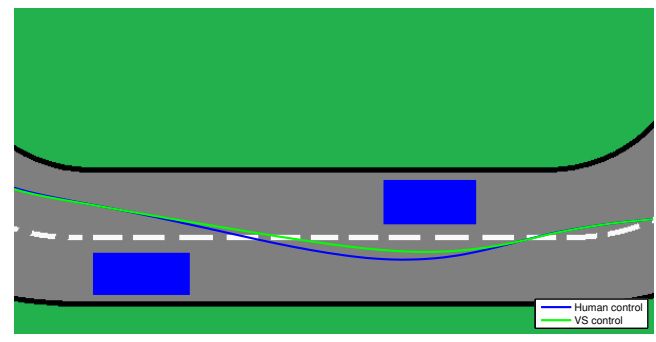

(a) HDB control driving route (in blue, hereinafter) and VS control driving route (in green, hereinafter), both in portion.

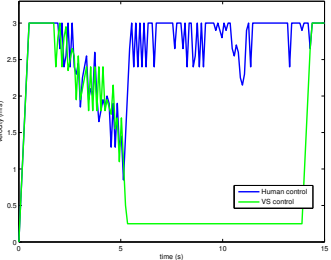

(b) Comparison of linear velocities.

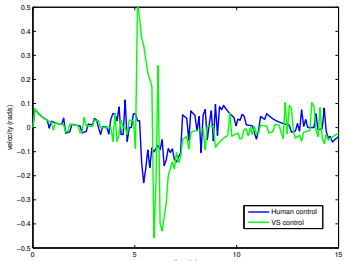

(c) Comparison of angular velocities.
Fig. 7. Comparison of performance in obstacle avoidance.

in a suitable interval. As a contrast, with the guidance of the VS controller, the angular velocity rapidly flipped with peak values considerably larger than those calculated by HDB controller. The experimental result proved HDB controller available and feasible with the cooperation of DWA method and a predefined referential path, while reducing the demand of deceleration in comparison with the VS controller.

\section{Danger Behaviors Correction}

In this experimental section, the performance of our methodology is examined in the circumstance where human drivers adopt danger behaviors while driving. For the purpose of representing the danger behaviors, the referential path is adjusted according to the evasive trajectory presented in the Section III-C. Firstly we postpone the turning of the path before bypassing an obstacle to simulate the delayed steering decision of human drivers. Another danger behavior is presented as a wrong turning which leads to a potential thread of collision. Both adjustments of the referential path are presented in the Figure 8(a).

The experimental result of the route, also presented in the Figure 8(a), illustrated that the robot successfully avoided the potential danger of collision with the application of HDB controller and VS-DWA method. The route taken by the robot applying the HDB controller held a safe distance from the obstacles and simultaneously remained smooth and stable from the perspective of the reference frame. In addition, the Figure 8(b) reclaimed the maintenance of linear velocity during the bypass period, as is mentioned in the Section IVB. Although a larger interval of angular velocity is observed in the Figure 8(c), in comparison with the result in the Figure 7(c), the peaks during the first period of steering is decreased, and a smooth passing manoeuvre is consequently guaranteed.

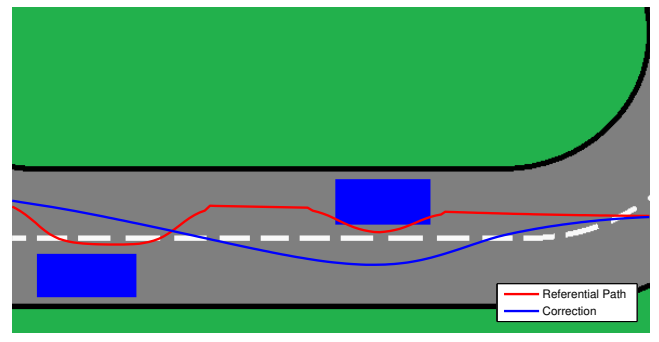

(a) Referential path with danger behaviors (in red) and corrected route by HDB controller (in blue), both in portion.

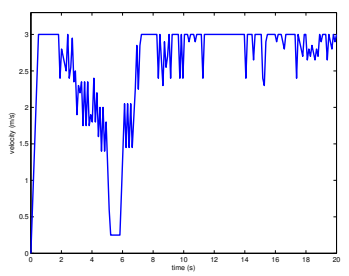

(b) Linear velocity calculated by (c) Angular velocity calculated by HDB controller.

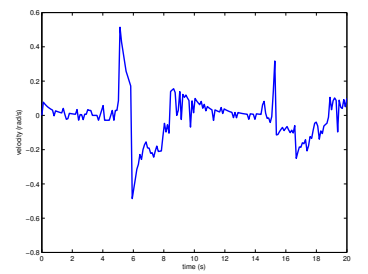

HDB controller.
Fig. 8. Result of danger behaviors correction performed by HDB controller.

In the following experiment, we took a step further to enlarge excessively the hazard of danger driving behavior by canceling the turning of the path before passing an obstacle. In such circumstance the obstacle occupies and blocks completely the referential pas as presented in the Figure 9(a).

The results presented in the Figure 9(a) revealed again a smooth and stable trajectory from the perspective of the reference frame with the manoeuvre of obstacle avoidance. The maintenance of linear velocity during the bypass period is also observed in such circumstance as illustrated in the Figure 9(b). The major concern in this part of experiment lies in the result of the angular velocity showed in the Figure 9(c). In comparison with the results from the previous experiments in the Figure 7(c) and 8(c), the angular velocity by HDB controller in such circumstance revealed a smooth process of steering with minor values of the angular velocity and fewer peaks. Due to the curve characteristics of the referential path, we conclude that the quality of the referential path influences considerably the behavior of HDB controller, thus affecting consequently the outcome of the practical control of the robot.

\section{CONCLUSION}

In this paper we have presented an approach of modeling the behaviors and decisions of human drivers, which has established the foundation of a simplified control pattern of human drivers' behaviors. With the application of DWA providing the validation of the linear and angular velocities of the vehicle, potential dangerous behaviors of human drivers have been refrained and corrected. Safety of driving in such circumstance has been therefore guaranteed. 


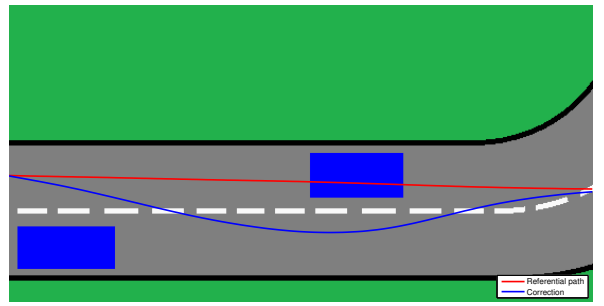

(a) Referential path with inevitable collision (in red) and corrected route by HDB controller (in blue), both in portion.

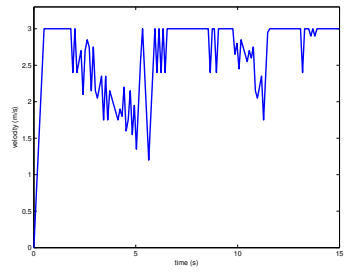

(b) Linear velocity calculated by (c) Angular velocity calculated by HDB controller.

Fig. 9. Result of danger behaviors correction performed by HDB controller.

In comparison with the experimental results of VS controller, our control pattern with the focus on human-vehicle interaction have proved its superiority in maintaining the smoothness and stability of the obstacle passing manoeuvre. Control strategies determined by human drivers, or in our approach by HDB controller, with both absolute values and smoothness of the control variables being promising, have also been confirmed as practical and applicable on actual intelligent vehicles.

Our approach has yet called for quite a few further progresses. The most apparent shortcoming of our design is the indispensable predefinition of the referential path or trajectory. A real-time calculation to achieve the referential trajectory from the perspective of human drivers, and a reliable localisation method to guarantee the acquisition of the position of the vehicle, are consequently desired. The characteristics of the referential path has also revealed its significance to the performance of the HDB controller. Therefore. These current deficiencies will point out the direction of our future research.

\section{REFERENCES}

[1] R. Ghandour, A. C. Victorino, A. Charara, and A. Lechner, "Risk indicators evaluation based on anticipated vehicle dynamics parameters," IEEE Intelligent Systems, pp. 68 - 73, 2012.

[2] F. Lotz, "System architectures for automated vehicle guidance concepts," in Automotive Systems Engineering. Springer, 2013, pp. 3961.

[3] S. Glaser, L. Nouveliere, and B. Lusetti, "Speed limitation based on an advanced curve warning system," in Intelligent Vehicles Symposium, 2007 IEEE. IEEE, 2007, pp. 686-691.

[4] A. Tang and A. Yip, "Collision avoidance timing analysis of dsrcbased vehicles," Accident Analysis \& Prevention, vol. 42, no. 1, pp. 182-195, 2010.

[5] W. Wang, W. Zhang, H. Guo, H. Bubb, and K. Ikeuchi, "A safety-based approaching behavioural model with various driving characteristics," Transportation research part C: emerging technologies, vol. 19, no. 6, pp. 1202-1214, 2011.
[6] T. Toledo, H. N. Koutsopoulos, and M. Ben-Akiva, "Integrated driving behavior modeling," Transportation Research Part C: Emerging Technologies, vol. 15, no. 2, pp. 96-112, 2007.

[7] — "Estimation of an integrated driving behavior model," Transportation Research Part C: Emerging Technologies, vol. 17, no. 4, pp. 365-380, 2009.

[8] R. Isermann, R. Mannale, and K. Schmitt, "Collision-avoidance systems proreta: Situation analysis and intervention control," Control Engineering Practice, 2012.

[9] A. Vahidi and A. Eskandarian, "Research advances in intelligent collision avoidance and adaptive cruise control," Intelligent Transportation Systems, IEEE Transactions on, vol. 4, no. 3, pp. 143-153, 2003.

[10] K. Suzuki and H. Jansson, "An analysis of drivers steering behaviour during auditory or haptic warnings for the designing of lane departure warning system," JSAE review, vol. 24, no. 1, pp. 65-70, 2003.

[11] M. Tsogas, A. Polychronopoulos, and A. Amditis, "Using digital maps to enhance lane keeping support systems," in Intelligent Vehicles Symposium, 2007 IEEE. IEEE, 2007, pp. 148-153.

[12] N. Minoiu Enache, M. Netto, S. Mammar, and B. Lusetti, "Driver steering assistance for lane departure avoidance," Control engineering practice, vol. 17, no. 6, pp. 642-651, 2009.

[13] P. F. Hokayem and M. W. Spong, "Bilateral teleoperation: An historical survey," Automatica, vol. 42, no. 12, pp. 2035-2057, 2006.

[14] A. Theriault, M. Nagurka, and M. Johnson, "Design and development of an affordable haptic robot with force-feedback and compliant actuation to improve therapy for patients with severe hemiparesis," 2013.

[15] P. Renon, C. Yang, H. Ma, and R. Cui, "Haptic interaction between human and virtual icub robot using novint falcon with chai3d and matlab," in Control Conference (CCC), 2013 32nd Chinese. IEEE, 2013, pp. 6045-6050.

[16] D. Fox, W. Burgard, and S. Thrun, "The dynamic window approach to collision avoidance," Robotics \& Automation Magazine, IEEE, vol. 4, no. 1, pp. 23-33, 1997.

[17] D. A. Lima and G. A. Pereira, "Navigation of an autonomous car using vector fields and the dynamic window approach," Journal of Control, Automation and Electrical Systems, pp. 1-11, 2013. [Online]. Available: http://dx.doi.org/10.1007/s40313-013-0006-5

[18] A. Cherubini, F. Chaumette, and G. Oriolo, "Visual servoing for path reaching with nonholonomic robots," Robotica, vol. 29, pp. 1037-1048, 12 2011. [Online]. Available: http://journals.cambridge. org/article_S0263574711000221

[19] A. D. Luca, G. Oriolo, A. De, and C. Samson, Robot Motion Planning and Control. Springer Berlin / Heidelberg, 1998, vol. 229, ch. Feedback Control Of A Nonholonomic Car-Like Robot, pp. 171-253.

[20] K. Rebai, O. Azouaoui, M. Benmami, and A. Larabi, "Car-like robot navigation at high speed," in Proceedings of the IEEE International Conference on Robotics and Biomimetics, 2007, pp. 2053-2057.

[21] K. Arras, J. Persson, N. Tomatis, and R. Siegwart, "Real-time obstacle avoidance for polygonal robots with a reduced dynamic window," in Proceedings of the IEEE International Conference on Robotics and Automation, vol. 3, 2002, pp. 3050-3055.

[22] T. A. Ranney, "Models of driving behavior: a review of their evolution," Accident Analysis \& Prevention, vol. 26, no. 6, pp. 733-750, 1994.

[23] A. Cherubini, F. Chaumette, and G. Oriolo, "An image-based visual servoing scheme for following paths with nonholonomic mobile robots," in Control, Automation, Robotics and Vision, 2008. ICARCV 2008. 10th International Conference on. IEEE, 2008, pp. 108-113.

[24] C. Keller, T. Dang, H. Fritz, A. Joos, C. Rabe, and D. Gavrila, "Active pedestrian safety by automatic braking and evasive steering," Intelligent Transportation Systems, IEEE Transactions on, vol. 12, no. 4, pp. 1292-1304, 2011.

[25] A. Houenou, P. Bonnifait, V. Cherfaoui, and W. Yao, "Vehicle trajectory prediction based on motion model and maneuver recognition," in Intelligent Robots and Systems (IROS), 2013 IEEE/RSJ International Conference on, 2013, pp. 4363-4369. 